\title{
Type-2 Fuzzy Sets Made Simple
}

\author{
Jerry M. Mendel and Robert I. Bob John
}

\begin{abstract}
Type-2 fuzzy sets let us model and minimize the effects of uncertainties in rule-base fuzzy logic systems. However, they are difficult to understand for a variety of reasons which we enunciate. In this paper, we strive to overcome the difficulties by: 1) establishing a small set of terms that let us easily communicate about type-2 fuzzy sets and also let us define such sets very precisely, 2) presenting a new representation for type-2 fuzzy sets, and 3) using this new representation to derive formulas for union, intersection and complement of type-2 fuzzy sets without having to use the Extension Principle.
\end{abstract}

Index Terms-Type-2 fuzzy logic systems, type-2 fuzzy sets.

\section{INTRODUCTION}

$\mathbf{I}$ T IS KNOWN that type-2 fuzzy sets let us model and minimize the effects of uncertainties in rule-based fuzzy logic systems (FLSs), ${ }^{1}$ e.g., [33]. Unfortunately, type-2 fuzzy sets are more difficult to use and understand than are type- 1 fuzzy sets; hence, their use is not yet widespread. In this paper we make type-2 fuzzy sets easy to use and understand in the hope that they will be widely used.

There are (at least) four sources of uncertainties in type-1 FLSs: (1) The meanings of the words that are used in the antecedents and consequents of rules can be uncertain (words mean different things to different people). (2) Consequents may have a histogram of values associated with them, especially when knowledge is extracted from a group of experts who do not all agree. (3) Measurements that activate a type-1 FLS may be noisy and therefore uncertain. (4) The data that are used to tune the parameters of a type-1 FLS may also be noisy. All of these uncertainties translate into uncertainties about fuzzy set membership functions. Type-1 fuzzy sets are not able to directly model such uncertainties because their membership functions are totally crisp. On the other hand, type-2 fuzzy sets are able to model such uncertainties because their membership functions are themselves fuzzy. Membership functions of type-1 fuzzy sets are two-dimensional, whereas membership functions of type-2 fuzzy sets are three-dimensional. It is the new third-dimension of

Manuscript received January 17, 2001; revised August 6, 2001 and September 6, 2001.

J. M. Mendel is with the Integrated Media Systems Center, University of Southern California, Los Angeles, CA 90089-2564 USA (e-mail: mendel@sipi.usc.edu).

R. I. B. John is with the Centre for Computational Intelligence, Department of Computer Science, Faculty of Computing Sciences and Engineering, De Montfort University, Leicester LE1 9BH, U.K. (e-mail: rij@dmu.ac.ak).

Publisher Item Identifier S 1063-6706(02)02960-0.

${ }^{1}$ The effects of uncertainties can be minimized by optimizing the parameters of the type-2 fuzzy sets during a training process. The additional parameters of type-2 fuzzy sets over those in type-1 fuzzy sets provide the former with additional design degrees of freedom that make it possible to minimize the effects of uncertainties. type-2 fuzzy sets that provides additional degrees of freedom that make it possible to directly model uncertainties.

Type-2 fuzzy sets are difficult to understand and use because: (1) the three-dimensional nature of type-2 fuzzy sets makes them very difficult to draw; (2) there is no simple collection of well-defined terms that let us effectively communicate about type-2 fuzzy sets, and to then be mathematically precise about them (terms do exist but have not been precisely defined ${ }^{2}$ ); (3) derivations of the formulas for the union, intersection, and complement of type-2 fuzzy sets all rely on using Zadeh's Extension Principle [44], which in itself is a difficult concept (especially for newcomers to FL) and is somewhat ad hoc, so that deriving things using it may be considered problematic; and, (4) using type-2 fuzzy sets is computationally more complicated than using type-1 fuzzy sets. In this paper, we focus on overcoming difficulties $1-3$, because doing so makes type- 2 fuzzy sets easy to use and understand. Difficulty 4 is the price one must pay for achieving better performance in the face of uncertainties, and is analogous to using probability rather than determinism. ${ }^{3}$ We only touch on it very briefly in this paper.

Even in the face of these difficulties, type-2 fuzzy sets and FLSs have already been used for (this list is in alphabetical order by application):

classification of coded video streams [29], co-channel interference elimination from nonlinear time-varying communication channels [28], connection admission control [30], control of mobile robots [42], decision making [43], [2], equalization of nonlinear fading channels [20], [32], [25], [27], extracting knowledge from questionnaire surveys [15], [30], forecasting of time-series [17], [32], [26], function approximation [15], learning linguistic membership grades [12], pre-processing radiographic images [13], relational databases [3], solving fuzzy relation equations [41], and transport scheduling [10].

They seem to be applicable when: (1) the data-generating system is known to be time-varying but the mathematical description of the time-variability is unknown (e.g., as in mobile communications); (2) measurement noise is nonstationary and the mathematical description of the nonstationarity is unknown (e.g., as in a time-varying SNR); (3) features in a pattern recognition application have statistical attributes that are nonstationary and the mathematical descriptions of the nonstationarities are unknown; (4) knowledge is mined from a group of experts using questionnaires that involve uncertain words; and (5) linguistic terms are used that have a nonmeasurable domain.

\footnotetext{
${ }^{2}$ This has been rectified in [33, Ch. 3]; however, it has not been rectified in any journal article.

${ }^{3}$ Free software is available on the Internet for implementing and designing type-2 FLSs at the following URL: http://sipi.usc.edu/ mendel/software.
} 
Before providing a summary of the coverage of this paper, we provide a brief history of type-2 fuzzy sets and FLSs. [44] introduced the concept of a type-2 fuzzy set as an extension of an ordinary fuzzy set, i.e., a type-1 fuzzy set. [34] studied the set theoretic operations of type-2 fuzzy sets and properties of membership grades of such sets; they also examined type- 2 fuzzy sets under the operations of algebraic product and algebraic sum [35]. [37] provided more detail about the algebraic structure of type-2 fuzzy sets. [14], [15], Karnik and Mendel extended the works of Mizumoto and Tanaka and obtained practical algorithms for performing union, intersection, and complement for type-2 fuzzy sets. They also developed the concept of the centroid of a type-2 fuzzy set and provided a practical algorithm for computing it for interval type-2 fuzzy sets [15], [19]. Dubois and Prade [4]-[6] discussed fuzzy valued logic and gave a formula for the composition of type-2 relations as an extension of the type-1 sup-star composition; but their formula is only for the minimum $t$-norm. A general formula for the extended sup-star composition of type-2 relations was given by [15], [16], and [20]. Based on this formula, [14]-[16] and [20] established a complete type-2 FLS theory. [8] studied rules and interval sets for higher-than-type-1 FL. [23], [24], [26] developed a complete theory for interval type-2 FLSs. They did this for different kinds of fuzzifiers, and showed how such FLSs can be designed, i.e., how the free parameters within interval type-2 FLSs can be tuned using training data. [12] developed a type-2 learning system that used training data to learn the membership grades of a type- 2 fuzzy system. For additional discussions on the use of interval sets in fuzzy logic, see [8], [38], [40], [7], [22], [36], [31], [21], and [1]. There are also some articles about type-2 fuzzy sets that have appeared in the Japanese literature, but are only in Japanese. Two examples are [9] and [39].

In Section II, we define a small set of terms that let us easily communicate about type-2 fuzzy sets, and let us define such sets in a mathematically precise way. One of these terms-footprint of uncertainty-enables us to graphically depict type-2 fuzzy sets in two-dimensions. In Section III, we provide a new representation for general type-2 fuzzy sets, one that re-expresses them in terms of much simpler type-2 fuzzy sets. In Section IV, we apply this new representation to the derivation of formulas for the union, intersection, and complement of type-2 fuzzy sets without having to use the Extension Principle. In Section V, we draw conclusions.

\section{TYPE-2 FuZZY SETS: DEFINITIONS}

In this section, we define type-2 fuzzy sets and some important associated concepts. By doing this, we provide a simple collection of mathematically well-defined terms that will let us effectively communicate about type- 2 fuzzy sets. This material is used extensively in the rest of this paper.

Imagine blurring the type-1 membership function depicted in Fig. 1(a) by shifting the points on the triangle either to the left or to the right and not necessarily by the same amounts, as in Fig. 1(b). Then, at a specific value of $x$, say $x^{\prime}$, there no longer is a single value for the membership function $\left(u^{\prime}\right)$; instead, the membership function takes on values wherever the vertical line intersects the blur. Those values need not all be weighted the same; hence, we can assign an amplitude distribution to all of

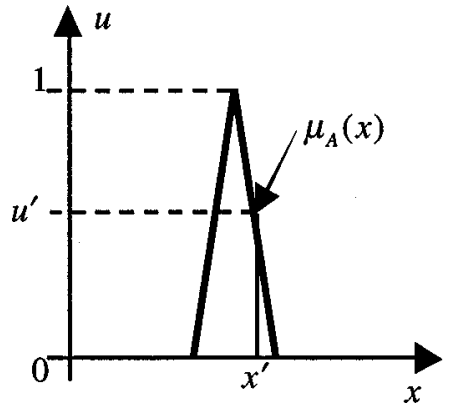

(a)

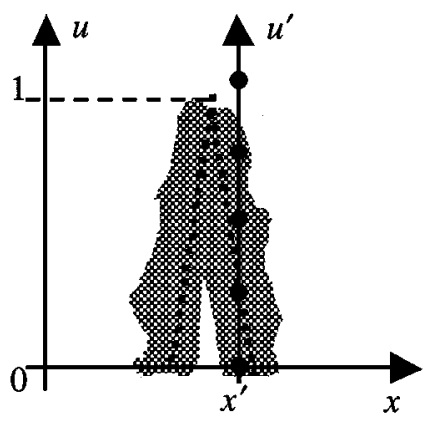

(b)

Fig. 1. (a) Type-1 membership function and (b) blurred type-1 membership function, including discretization at $x=x^{\prime}$.

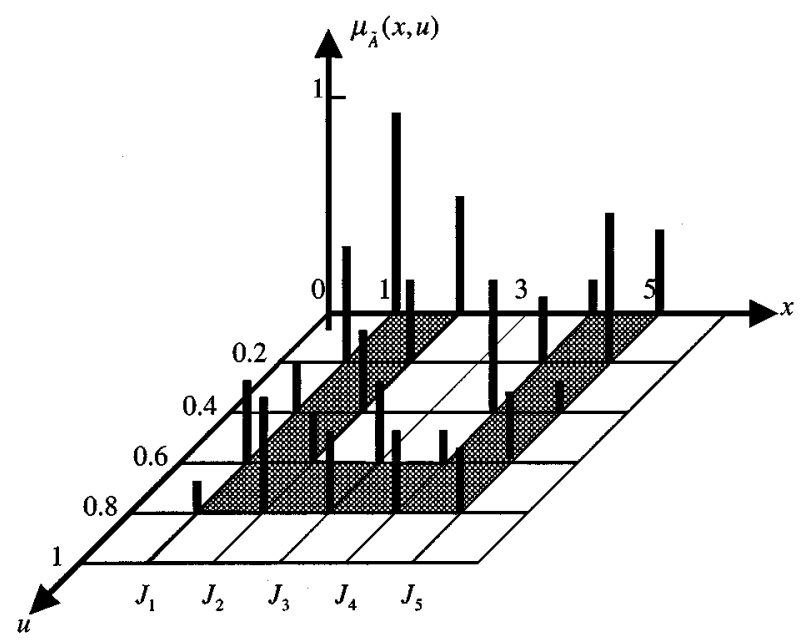

Fig. 2. Example of a type-2 membership function. The shaded area is the FOU.

those points. Doing this for all $x \in X$, we create a three-dimensional membership function-a type- 2 membership function-that characterizes a type-2 fuzzy set.

Definition 1: A type-2 fuzzy set, denoted $\tilde{A}$, is characterized by a type-2 membership function $\mu_{\tilde{A}}(x, u)$, where $x \in X$ and $u \in J_{x} \subseteq[0,1]$, i.e.,

$$
\tilde{A}=\left\{\left((x, u), \mu_{\tilde{A}}(x, u)\right) \mid \forall x \in X, \forall u \in J_{x} \subseteq[0,1]\right\}
$$

in which $0 \leq \mu_{\tilde{A}}(x, u) \leq 1 . \tilde{A}$ can also be expressed as

$$
\tilde{A}=\int_{x \in X} \int_{u \in J_{x}} \mu_{\tilde{A}}(x, u) /(x, u) \quad J_{x} \subseteq[0,1]
$$




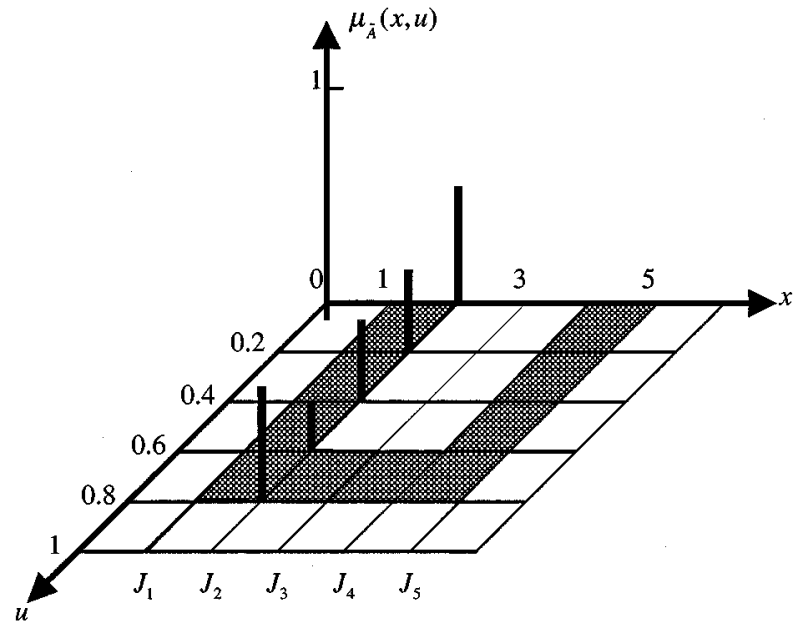

Fig. 3. Example of a vertical slice for the type-2 membership function depicted in Fig. 2.

where $\iint$ denotes union $^{4}$ over all admissible $x$ and $u$. For discrete universes of discourse $\int$ is replaced by $\sum$.

In Definition 1, the first restriction that $\forall u \in J_{x} \subseteq[0,1]$ is consistent with the type- 1 constraint that $0 \leq \mu_{A}(x) \leq 1$, i.e., when uncertainties disappear a type- 2 membership function must reduce to a type- 1 membership function, in which case the variable $u$ equals $\mu_{A}(x)^{5}$ and $0 \leq \mu_{A}(x) \leq 1$. The second restriction that $0 \leq \mu_{\tilde{A}}(x, u) \leq 1$ is consistent with the fact that the amplitudes of a membership function should lie between or be equal to 0 and 1 .

Example 1: Fig. 2 depicts $\mu_{\tilde{A}}(x, u)$ for $x$ and $u$ discrete. In particular, $X=\{1,2,3,4,5\}$ and $U=\{0,0.2,0.4,0.6,0.8\}$.

Definition 2: At each value of $x$, say $x=x^{\prime}$, the 2-D plane whose axes are $u$ and $\mu_{\tilde{A}}\left(x^{\prime}, u\right)$ is called a vertical slice of $\mu_{\tilde{A}}(x, u)$. A secondary membership function is a vertical slice of $\mu_{\tilde{A}}(x, u)$. It is $\mu_{\tilde{A}}\left(x=x^{\prime}, u\right)$ for $x \in X$ and $\forall u \in J_{x^{\prime}} \subseteq[0,1]$, i.e.,

$$
\mu_{\tilde{A}}\left(x=x^{\prime}, u\right) \equiv \mu_{\tilde{A}}\left(x^{\prime}\right)=\int_{u \in J_{x^{\prime}}} f_{x^{\prime}}(u) / u \quad J_{x^{\prime}} \subseteq[0,1]
$$

in which $0 \leq f_{x^{\prime}}(u) \leq 1$. Because $\forall x^{\prime} \in X$, we drop the prime notation on $\mu_{\tilde{A}}\left(x^{\prime}\right)$, and refer to $\mu_{\tilde{A}}(x)$ as a secondary membership function; it is a type-1 fuzzy set, which we also refer to as a secondary set.

Example 1 (Continued): The type-2 membership function that is depicted in Fig. 2 has five vertical slices associated with it. The one at $x=2$ is depicted in Fig. 3. The secondary membership function at $x=2$ is $\mu_{\tilde{A}}(2)=0.5 / 0+0.35 / 0.2+$ $0.35 / 0.4+0.2 / 0.6+0.5 / 0.8$.

Based on the concept of secondary sets, we can reinterpret a type-2 fuzzy set as the union (see footnote 4 ) of all secondary

${ }^{4}$ Recall that the union of two sets $A$ and $B$ is by definition another set that contains the elements in either $A$ or $B$. When we view each element of a type-2 fuzzy set as a subset, then the unions in (2) conform to the classical definition of union, since each element of that set is distinct. At a specific value of $x$ and $u$ only one term is activated in the union.

${ }^{5}$ In this case, the third dimension disappears. sets, i.e., using (3), we can re-express $\tilde{A}$ in a vertical-slice manner, as

$$
\tilde{A}=\left\{\left(x, \mu_{\tilde{A}}(x)\right) \mid \forall x \in X\right\}
$$

or, as

$$
\begin{aligned}
& \tilde{A}=\int_{x \in X} \mu_{\tilde{A}}(x) / x=\int_{x \in X}\left[\int_{u \in J_{x}} f_{x}(u) / u\right] / x \\
& J_{x} \subseteq[0,1] .
\end{aligned}
$$

Definition 3: The domain of a secondary membership function is called the primary membership of $x$. In (5), $J_{x}$ is the primary membership of $x$, where $J_{x} \subseteq[0,1]$ for $\forall x \in X$.

Definition 4: The amplitude of a secondary membership function is called a secondary grade. In $(5), f_{x}(u)$ is a secondary grade; in (1), $\mu_{\tilde{A}}\left(x^{\prime}, u^{\prime}\right)\left(x^{\prime} \in X, u^{\prime} \in J_{x^{\prime}}\right)$ is a secondary grade.

If $X$ and $J_{x}$ are both discrete (either by problem formulation, as in Example 1, or by discretization of continuous universes of discourse), then the right-most part of (5) can be expressed as

$$
\begin{aligned}
\tilde{A} & =\sum_{x \in X}\left[\sum_{u \in J_{x}} f_{x}(u) / u\right] / x \\
= & \sum_{i=1}^{N}\left[\sum_{u \in J_{x_{i}}} f_{x_{i}}(u) / u\right] / x_{i} \\
= & {\left[\sum_{k=1}^{M_{1}} f_{x_{1}}\left(u_{1 k}\right) / u_{1 k}\right] / x_{1}+\cdots } \\
& +\left[\sum_{k=1}^{M_{N}} f_{x_{N}}\left(u_{N k}\right) / u_{N k}\right] / x_{N} .
\end{aligned}
$$

In this equation, + also denotes union. Observe that $x$ has been discretized into $N$ values and at each of these values $u$ has been discretized into $M_{i}$ values. The discretization along each $u_{i k}$ does not have to be the same, which is why we have shown a different upper sum for each of the bracketed terms. If, however, the discretization along each $u_{i k}$ is the same, then $M_{1}=M_{2}=$ $\cdots=M_{N} \equiv M$.

Example 1 (Continued): In Fig. 2, the union of the five secondary membership functions at $x=1,2,3,4,5$ is $\mu_{\tilde{A}}(x, u)$. Observe that the primary memberships are

$$
\begin{aligned}
& J_{1}=J_{2}=J_{4}=J_{5}=\{0,0.2,0.4,0.6,0.8\} \quad \text { and } \\
& J_{3}=\{0.6,0.8\}
\end{aligned}
$$

and, we have only included values in $J_{3}$ for which $\mu_{\tilde{A}}(x, u) \neq$ 0 . Each of the spikes in Fig. 1 represents $\mu_{\tilde{A}}(x, u)$ at a specific $(x, u)$-pair, and its amplitude is a secondary grade.

Definition 5: Uncertainty in the primary memberships of a type-2 fuzzy set, $\tilde{A}$, consists of a bounded region that we call the footprint of uncertainty (FOU). It is the union of all primary memberships, i.e.,

$$
\operatorname{FOU}(\tilde{A})=\bigcup_{x \in X} J_{x}
$$

The shaded region in Fig. 1 is the FOU. Other examples of FOUs are given in Fig. 4. The term footprint of uncertainty is very useful, because it not only focuses our attention on the un- 
certainties inherent in a specific type- 2 membership function, whose shape is a direct consequence of the nature of these uncertainties, but it also provides a very convenient verbal description of the entire domain of support for all the secondary grades of a type-2 membership function. It also lets us depict a type-2 fuzzy set graphically in two-dimensions instead of three dimensions, and in so doing lets us overcome the first difficulty about type-2 fuzzy sets-their three-dimensional nature which makes them very difficult to draw. The shaded FOUs imply that there is a distribution that sits on top of it-the new third dimension of type-2 fuzzy sets. What that distribution looks like depends on the specific choice made for the secondary grades. When they all equal one, the resulting type- 2 fuzzy sets are called interval type-2 fuzzy sets. Such sets are the most widely used type- 2 fuzzy sets to date.

Definition 6: For discrete universes of discourse $X$ and $U$, an embedded type-2 set $\tilde{A}_{e}$ has $N$ elements, where $\tilde{A}_{e}$ contains exactly one element from $J_{x_{1}}, J_{x_{2}}, \ldots, J_{x_{N}}$, namely $u_{1}, u_{2}, \ldots, u_{N}$, each with its associated secondary grade, namely $f_{x_{1}}\left(u_{1}\right), f_{x_{2}}\left(u_{2}\right), \ldots, f_{x_{N}}\left(u_{N}\right)$, i.e.,

$$
\tilde{A}_{e}=\sum_{i=1}^{N}\left[f_{x_{i}}\left(u_{i}\right) / u_{i}\right] / x_{i} \quad u_{i} \in J_{x_{i}} \subseteq U=[0,1] .
$$

Set $\tilde{A}_{e}$ is embedded in $\tilde{A}$, and, there are a total ${ }^{6}$ of $\prod_{i=1}^{N} M_{i} \tilde{A}_{e}$.

Definition 7: For discrete universes of discourse $X$ and $U$, an embedded type-1 set $A_{e}$ has $N$ elements, one each from $J_{x_{1}}, J_{x_{2}}, \ldots, J_{x_{N}}$, namely $u_{1}, u_{2}, \ldots, u_{N}$, i.e.,

$$
A_{e}=\sum_{i=1}^{N} u_{i} / x_{i} \quad u_{i} \in J_{x_{i}} \subseteq U=[0,1] .
$$

Set $A_{e}$ is the union of all the primary memberships of set $\tilde{A}_{e}$ in (8), and, there are a total of $\prod_{i=1}^{N} M_{i} A_{e}$.

Example 2: Fig. 5 depicts one of the possible $1250 \mathrm{em}-$ bedded type- 2 sets for the type- 2 membership function that is depicted in Fig. 2. Observe that the embedded type-1 set that is associated with this embedded type-2 set is $A_{e}=0 / 1+0.4 / 2+0.8 / 3+0.8 / 4+0.4 / 5$.

Definition 8: A type-1 fuzzy set can also be expressed as a type-2 fuzzy set. Its type-2 representation is $\left(1 / \mu_{F}(x)\right) / x$ or $1 / \mu_{F}(x), \forall_{x} \in X$, for short. The notation $1 / \mu_{F}(x)$ means that the secondary membership function has only one value in its domain, namely the primary membership $\mu_{F}(x)$, at which the secondary grade equals 1 .

\section{A New RePResentation FOR TyPe-2 FuZZy SETS}

So far we have emphasized the vertical-slice representation (decomposition) of a type-2 fuzzy set as given in (5). In this section, we provide a new (canonical) representation for a type-2 fuzzy set that is in terms of so-called wavy slices. This representation makes very heavy use of embedded type-2 fuzzy sets (Definition 6). Before we state and prove this new representation, we state and prove the following preliminary result:

${ }^{6}$ For continuous type- 2 fuzzy sets, there are an uncountable number of embedded type-2 fuzzy sets, and this concept is not very useful.

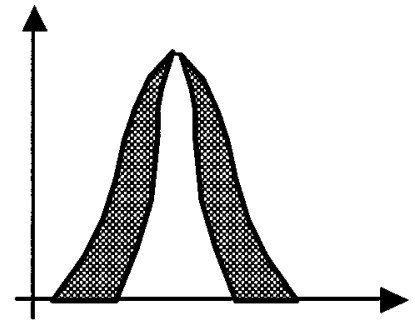

(a)

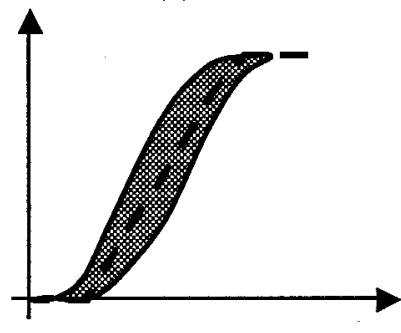

(c)

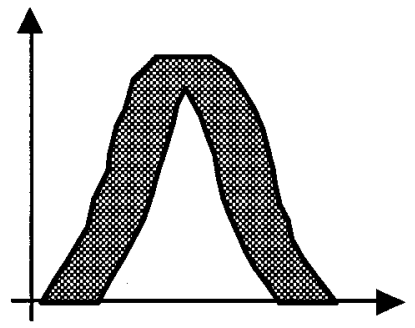

(b)

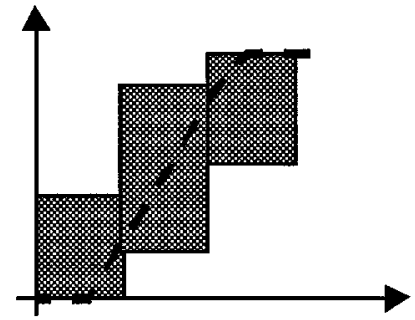

(d)
Fig. 4. FOUs. (a) Gaussian MF with uncertain standard deviation. (b) Gaussian MF with uncertain mean. (c) Sigmoidal MF with inflection uncertainties. (d) Granulated sigmoidal MF with granulation uncertainties.

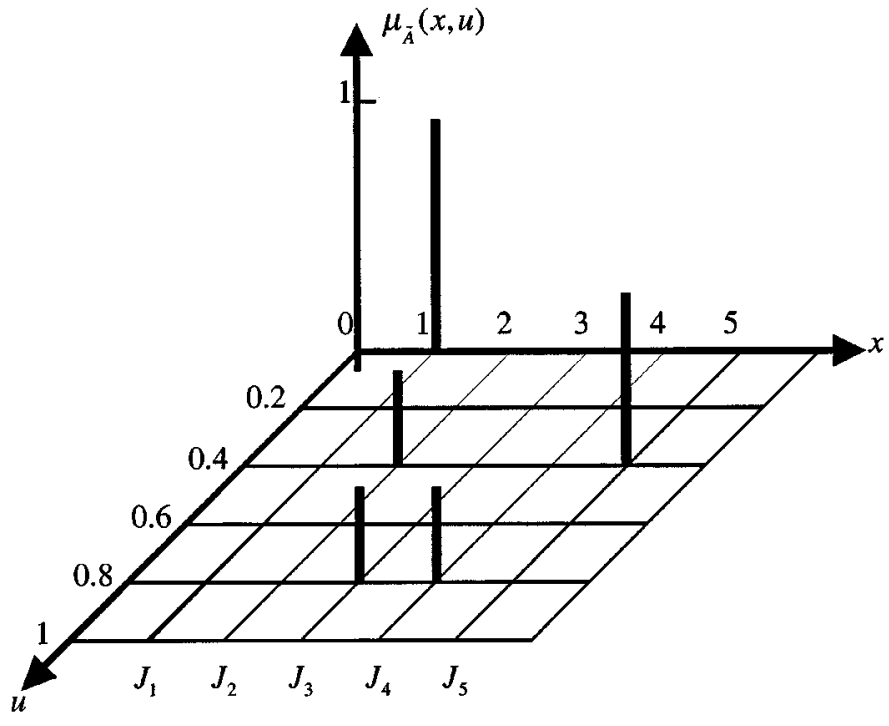

Fig. 5. Example of an embedded type-2 set associated with the type-2 membership function depicted in Fig. 2.

Theorem 1: Consider the general FOU that is depicted in Fig. 6 . We call each point along the $u_{i}$ line a node. Each node along the $u_{i}$-axis is contained in exactly

$$
n_{l}=\prod_{j=1, j \neq l}^{N} M_{j}
$$

embedded (type-2 or type-1) sets, where $l=1,2, \ldots, N$.

Proof: All embedded sets start with an element along the $u_{1}$-axis, and each element along that axis fans out into exactly $\prod_{j=2}^{N} M_{j}$ embedded sets. Note that

$$
\prod_{j=2}^{N} M_{j}=\prod_{j=1, j \neq 1}^{N} M_{j} \equiv n_{1} .
$$




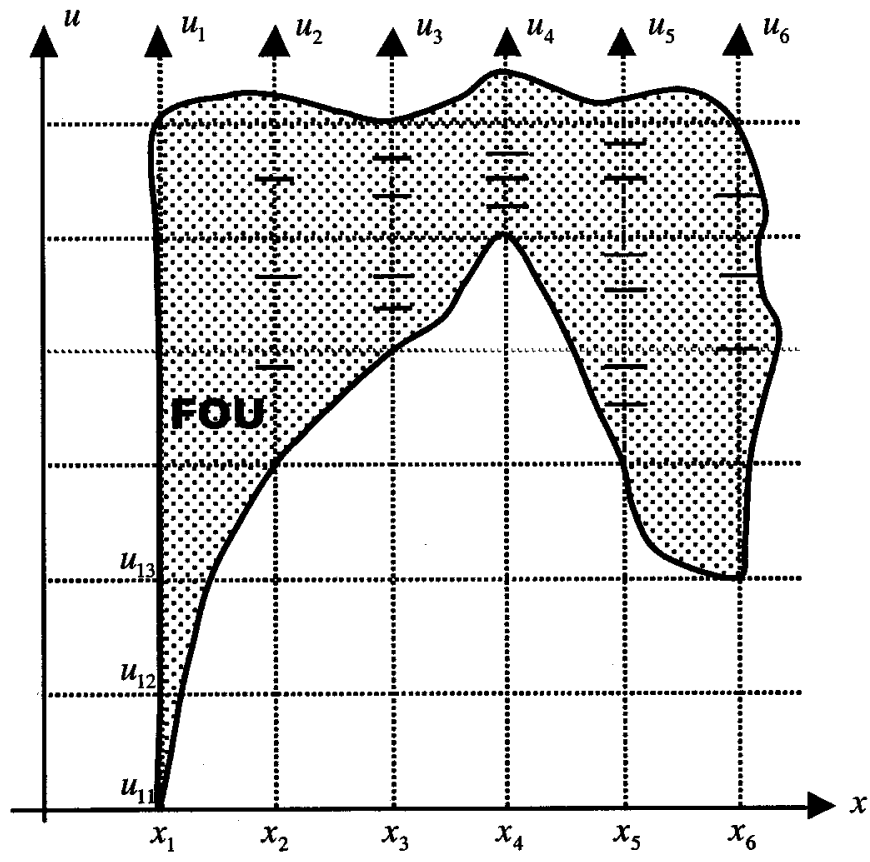

Fig. 6. Example of a FOU on discretized $x$ - and $u$-axes. Here $N=6$.

Next, consider elements along the $u_{2}$-axis. The $M_{1}$ elements along the $u_{1}$-axis fan into all the elements along the $u_{2}$-axis after which each element along the $u_{2}$-axis fans out into exactly $\prod_{j=3}^{N} M_{j}$ embedded sets. This means that there are a total of $M_{1} \prod_{j=3}^{N} M_{j}$ embedded sets for each node along the $u_{2}$-axis. Note that

$$
M_{1} \prod_{j=3}^{N} M_{j}=\prod_{j=1, j \neq 2}^{N} M_{j} \equiv n_{2} .
$$

Continuing in this way for the $u_{3^{-}}, \ldots$, and $u_{N^{-}}$-axes, we obtain the result in (10).

Next, we present a new decomposition for a type-2 fuzzy set that we refer to as a Representation Theorem.

Theorem 2 (Representation Theorem): Let $\tilde{A}_{e}^{j}$ denote the $j$ th type- 2 embedded set for type- 2 fuzzy set $\tilde{A}$, i.e.,

$$
\tilde{A}_{e}^{j} \equiv\left\{\left(u_{i}^{j}, f_{x_{i}}\left(u_{i}^{j}\right)\right), i=1, \ldots, N\right\}
$$

where (see Definition 6 and Fig. 6)

$$
u_{i}^{j} \in\left\{u_{i k}, k=1, \ldots, M_{i}\right\} .
$$

Then $\tilde{A}$ can be represented as the union of its type- 2 embedded sets, i.e.,

$$
\tilde{A}=\sum_{j=1}^{n} \tilde{A}_{e}^{j}
$$

where $^{7}$

$$
n \equiv \prod_{i=1}^{N} M_{i}
$$

${ }^{7}$ The summation in (15) is still a classical union. Duplicated elements (there will be many of them because the embedded type- 2 fuzzy sets contain many duplicated elements) only count once, as in any union.

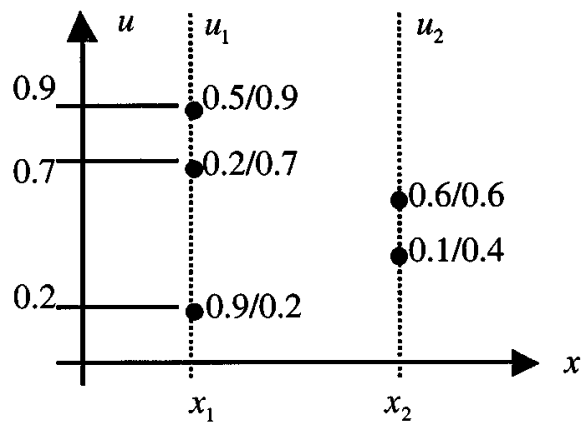

(a)

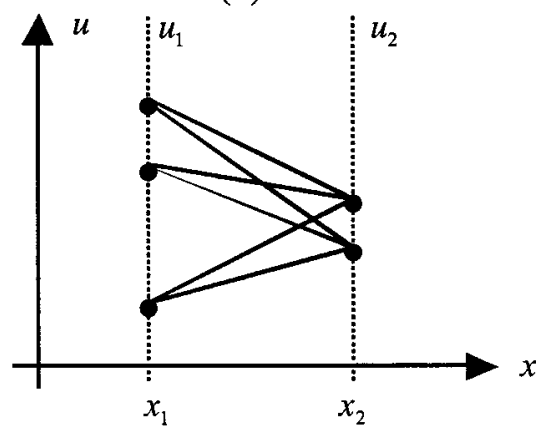

(b)

Fig. 7. (a) Vertical-slice representation for $\tilde{A}$ in (19). (b) Six embedded type-2 fuzzy sets, each connected by a line from $x_{1}$ to $x_{2}$.

Proof: We prove this theorem by demonstrating that (6) can be re-expressed so that it contains exactly all of the terms on the right-hand side of (15). Theorem 1 is the key to doing this. What we do is to repeat term 1 in (6) $n_{1}$ times, term 2 in (6) $n_{2}$ times, ..., and term $N$ in (6) $n_{N}$ times [using the fact, e.g., that $\tilde{A}_{i}+\tilde{A}_{i}=\tilde{A}_{i}$ (where + denotes union)], i.e.,

$$
\begin{aligned}
\tilde{A}=\sum_{i=1}^{n_{1}}\{[ & {\left.\left[\sum_{k=1}^{M_{1}} f_{x_{1}}\left(u_{1 k}\right) / u_{1 k}\right] / x_{1}\right\}+\cdots } \\
& +\sum_{i=1}^{n_{N}}\left\{\left[\sum_{k=1}^{M_{N}} f_{x_{N}}\left(u_{N k}\right) / u_{N k}\right] / x_{N}\right\} .
\end{aligned}
$$

We must now demonstrate that (17) can be reorganized as in (15). We do this by proving that (17) has exactly the same number of terms (elements) as does (15). The actual construction of the $\tilde{A}_{e}^{j}$ is discussed below in Comment 1.

Note that, according to Definition 6, each $\tilde{A}_{e}^{j}$ has exactly $N$ elements. Hence, $\tilde{A}$ in (15) has exactly $n N=N \times \prod_{i=1}^{N} M_{i}$ elements (many of which are duplicated). In (17), $\tilde{A}$ has $n_{1} M_{1}+$ $n_{2} M_{2}+\cdots+n_{N} M_{N}$ elements; but, from (10), it follows that:

$$
\begin{aligned}
& n_{1} M_{1}+n_{2} M_{2}+\cdots+n_{N} M_{N} \\
& \quad=\prod_{i=1}^{N} M_{i}+\prod_{i=1}^{N} M_{i}+\cdots+\prod_{i=1}^{N} M_{i} \\
& \quad=N \times \prod_{i=1}^{N} M_{i}
\end{aligned}
$$

which proves that $\tilde{A}$ in (17) has exactly the same number of elements as $\tilde{A}$ in (15).

Comment 1: In order to implement (15), one needs a constructive method for specifying each $\tilde{A}_{e}^{j}$. Note that $j$ is the 
solution to the following combinatorial assignment problem: Determine all possible combinations $\left(a_{1}, a_{2}, \ldots, a_{N}\right)$ such that $\left(u_{1 a_{1}}, u_{2 a_{2}}, \ldots, u_{N a_{N}}\right) \rightarrow j$, where $a_{1} \in$ $\left\{1,2, \ldots, M_{1}\right\}, a_{2} \in\left\{1,2, \ldots, M_{2}\right\}, \ldots, a_{N} \quad \in$ $\left\{1,2, \ldots, M_{N}\right\}$, and $j=1,2, \ldots, \prod_{i=1}^{N} M_{i}$. A computer program can be written to map $\left(a_{1}, a_{2}, \ldots, a_{N}\right)$ into $j$. For the purposes of this paper, we do not need such a program, because we only use (15) for theoretical derivations and not for computational purposes. Its use for computational purposes would be terribly inefficient since it contains an enormous amount of redundancy.

Comment 2: Theorem 2 expresses $\tilde{A}$ as a union of simpler type-2 fuzzy sets, the $\tilde{A}_{e}^{j}$. They are simpler because their secondary membership functions are singletons. Whereas (5) is a vertical slice representation of $\tilde{A},(15)$ is a wavy slice representation of $\tilde{A}$.

Example 3: Consider the following type-2 fuzzy set:

$$
\begin{aligned}
\tilde{A}=(0.5 / 0.9) / x_{1}+ & (0.2 / 0.7) / x_{1}+(0.9 / 0.2) / x_{1} \\
& +(0.6 / 0.6) / x_{2}+(0.1 / 0.4) / x_{2} .
\end{aligned}
$$

The vertical-slice representation of $\tilde{A}$ is depicted in Fig. 7 . Observe that $M_{1}^{A}=3, M_{2}^{A}=2$, and $n_{A}=M_{1}^{A} M_{2}^{A}=6$. Hence, there are six embedded type- 2 sets, namely

$$
\begin{aligned}
\tilde{A}_{e}^{1} & =(0.5 / 0.9) / x_{1}+(0.6 / 0.6) / x_{2} \\
\tilde{A}_{e}^{2} & =(0.5 / 0.9) / x_{1}+(0.1 / 0.4) / x_{2} \\
\tilde{A}_{e}^{3} & =(0.2 / 0.7) / x_{1}+(0.6 / 0.6) / x_{2} \\
\tilde{A}_{e}^{4} & =(0.2 / 0.7) / x_{1}+(0.1 / 0.4) / x_{2} \\
\tilde{A}_{e}^{5} & =(0.9 / 0.2) / x_{1}+(0.6 / 0.6) / x_{2} \\
\tilde{A}_{e}^{6} & =(0.9 / 0.2) / x_{1}+(0.1 / 0.4) / x_{2}
\end{aligned}
$$

It is very easy to see (refer to footnote 3 ) that $\tilde{A}=$ $\sum_{j=1}^{6} \tilde{A}_{e}^{j}$.

\section{APPLiCATIONS}

In this section, we apply the Representation Theorem to the derivation of formulas for union, intersection and complement of type-2 fuzzy sets, without having to use the Extension Principle.

\section{A. Union of Type-2 Fuzzy Sets}

Consider two type-2 fuzzy sets $\tilde{A}$ and $\tilde{B}$, where

$$
\begin{array}{r}
\tilde{A}=\sum_{x \in X} \mu_{\tilde{A}}(x) /(x) \sum_{x \in X}\left[\sum_{u \in J_{x}^{u}} f_{x}(u) / u\right] / x \\
J_{x}^{u} \subseteq[0,1]
\end{array}
$$

and

$$
\begin{array}{r}
\tilde{B}=\sum_{x \in X} \mu_{\tilde{B}}(x) / x=\sum_{x \in X}\left[\sum_{w \in J_{x}^{w}} g_{x}(w) / w\right] / x \\
J_{x}^{w} \subseteq[0,1] .
\end{array}
$$

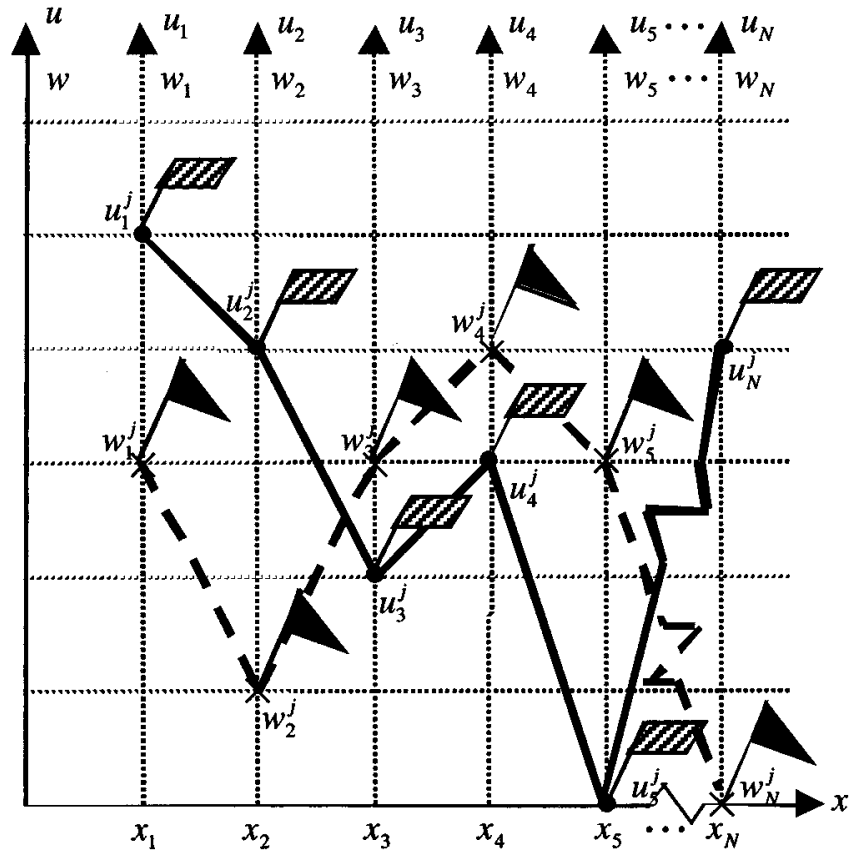

Fig. 8. Two representative embedded type-2 fuzzy sets. The filled circles and rectangular flags denote the primary memberships and secondary grades for $\tilde{A}_{e}^{i}$, whereas the crosses and triangular flags denote the primary memberships and secondary grades for $\tilde{B}_{e}^{i}$. The solid and dashed curves are associated with the embedded type-1 fuzzy sets, $A_{e}^{j}$ and $B_{e}^{i}$, respectively.

From Representation Theorem 2, it follows that: ${ }^{8}$

$$
\tilde{A} \cup \tilde{B}=\sum_{j=1}^{n_{A}} \tilde{A}_{e}^{j} \cup \sum_{i=1}^{n_{B}} \tilde{B}_{e}^{i}=\sum_{j=1}^{n_{A}} \sum_{i=1}^{n_{B}} \tilde{A}_{e}^{j} \cup \tilde{B}_{e}^{i} .
$$

This demonstrates that to evaluate $\tilde{A} \cup \tilde{B}$ we need to evaluate the union of type- 2 embedded sets, i.e., we need to evaluate $\tilde{A}_{e}^{j} \cup \tilde{B}_{e}^{i}(\forall i, j)$, a calculation to which we turn next.

Two representative embedded type-2 fuzzy sets are depicted in Fig. 8. Each embedded type-2 set has only one node on its $u_{l^{-}}$or $w_{k}$-axes. The rectangular and triangular flags denote the secondary grades at each node, and are merely meant as a pneumonic for those grades.

Next, recall (as explained in Section II) that a type-1 fuzzy set can be interpreted as a type-2 fuzzy set all of whose secondary grades equal unity (i.e., all flags equal 1). In fact, a type-1 fuzzy set is an instance of a type-2 fuzzy set. It is a crisp version of a type-2 fuzzy set. Given that this is the case, it seems sensible to consider using the type-1 definitions for union, intersection, and complement as a starting point and generalizing them to a fuzzy type- 1 fuzzy set-a type-2 fuzzy set. Next, we show that by taking this approach we do not directly need to use, or make any reference to, the Extension Principle.

\footnotetext{
${ }^{8}$ This equation involves summations and union signs. As in the type- 1 case, where this mixed notation is used, the summation sign is simply shorthand for lots of + signs. The + indicates the union between members of a set, whereas the union sign represents the union of the sets themselves. Hence, by using both the summation and union signs, we are able to distinguish between the union of sets versus the union of members within a set.
} 
Recall, from Definition 7, that $\tilde{A}_{e}^{j}$ and $\tilde{B}_{e}^{i}$ are the embedded type-1 fuzzy sets that are associated with $\tilde{A}_{e}^{j}$ and $\tilde{B}_{e}^{i}$, respectively. In the type- 1 case

$$
\begin{aligned}
A_{e}^{j} \cup B_{e}^{i}=u_{1}^{j} \vee w_{1}^{i} / x_{1}+u_{2}^{j} \vee w_{2}^{i} / & x_{2}+\cdots \\
& +u_{N}^{j} \vee w_{N}^{i} / x_{N}
\end{aligned}
$$

which (see Definition 8) can be expressed as a type-2 set, as follows:

$$
\begin{aligned}
A_{e}^{j} \cup B_{e}^{i}=\left[1 / u_{1}^{j} \vee w_{1}^{i}\right] / x_{1}+ & {\left[1 / u_{2}^{j} \vee w_{2}^{i}\right] / x_{2}+\cdots } \\
+ & {\left[1 / u_{N}^{j} \vee w_{N}^{i}\right] / x_{N} . }
\end{aligned}
$$

Observe that in this representation, the "flag" (i.e., secondary grade) at each $u_{l}^{j} \vee w_{l}^{i}$ point is unity.

In the type- 2 case, where the flags start out being different at each $u_{l}^{j}$ and $w_{l}^{i}$ points, we need to keep track of them as we perform $\tilde{A}_{e}^{j} \cup \tilde{B}_{e}^{i}$. Call the flag (see Fig. 8, e.g., for $l=2$ ) at $u_{l}^{j}$, which occurs when $x=x_{l}, f_{x_{l}}\left(u_{l}^{j}\right)$, and the flag at $w_{l}^{i}$, which also occurs when $x=x_{l}, g_{x_{l}}\left(w_{l}^{i}\right)$. Let $h$ be some operation (defined below) on the two flags that produces a new flag that uniquely identifies $u_{l}^{j} \vee w_{l}^{i}$. Call this new flag $F_{x_{l}}\left(u_{l}^{j}, w_{l}^{i}\right)$, i.e.,

$$
F_{x_{l}}\left(u_{l}^{j}, w_{l}^{i}\right)=h\left[f_{x_{l}}\left(u_{l}^{j}\right), g_{x_{l}}\left(w_{l}^{i}\right)\right] .
$$

In analogy to (25), we now define $\tilde{A}_{e}^{j} \cup \tilde{B}_{e}^{i}$ as follows:

$$
\begin{aligned}
\tilde{A}_{e}^{j} \cup \tilde{B}_{e}^{i} \equiv\left[F_{x_{1}}\left(u_{1}^{j}, w_{1}^{i}\right) / u_{1}^{j} \vee w_{1}^{i}\right] / x_{1}+\cdots \\
+\left[F_{x_{N}}\left(u_{N}^{j}, w_{N}^{i}\right) / u_{N}^{j} \vee w_{N}^{i}\right] / x_{N} .
\end{aligned}
$$

This is a very plausible way to define the union of embedded type-2 fuzzy sets, since it reduces to our accepted type- 1 definition of union when all flags equal unity. It also establishes the constraint that $h(1,1)=1$, which forces the type-2 result in (27) to reduce to the type-1 result in (25) when all uncertainties disappear.

Next, we demonstrate that an appropriate choice for $h$ is a $t$-norm. We begin by requiring $h$ to have the following four properties:

1) $h(a, b)=h(b, a)$

2) $0 \leq h(a, b) \leq 1$

3) $h(1,1)=1$

4) $h(a, 0)=h(0, b)=0$.

Property 1) is intuitive, because it should not matter in which order we handle the flags $a$ and $b$. Property 2 ) is also intuitive, because $h(a, b)$ is another flag, which means that it is a secondary grade for a secondary membership function, and all secondary grades must be bounded according to Definition 1 . We have already justified Property 3 ). Property 4 ) involves flags that each have zero values. Such flags (which are perfectly permissible) allow secondary membership functions to have zero (i.e., vacuous) secondary grades at specific values of primary memberships. Property 4 requires that a vacuous flag in one fuzzy set must remain vacuous in any fuzzy set derived from it, which again seems plausible. It is now obvious, from the properties of a $t$-norm, that:
Lemma 1: Under the four properties just stated, $h$ is a $t$-norm.

Comment 3: No doubt, one could require a different Property 4, e.g., $h(a, 0)=a$ and $h(0, b)=b$, in which case $h$ would be a $t$-conorm. We find such a requirement for $h$ much less plausible than our Property 4, and so recommend choosing $h$ as a $t$-norm.

Comment 4: People already familiar with the Extension Principle will claim that (27) is exactly what would be obtained by applying the existing formula for the union of two type-2 fuzzy sets to $\widetilde{A}_{e}^{j}$ and $\tilde{B}_{e}^{i}$, and they would be correct (when $h$ is a $t$-norm). However, we have reached (27) without needing to know anything about the Extension Principle, and would have reached that point even if the Extension Principle didn't exist.

We are now ready to state the main result of this section.

Theorem 3: The union of two type-2 fuzzy sets $\tilde{A}$ and $\tilde{B}$ is given as

$$
\begin{gathered}
\tilde{A} \cup \tilde{B}=\sum_{j=1}^{n_{A}} \sum_{i=1}^{n_{B}}\left\{\left[F_{x_{1}}\left(u_{1}^{j}, w_{1}^{i}\right) / u_{1}^{j} \vee w_{1}^{i}\right] / x_{1}+\cdots\right. \\
\left.+\left[F_{x_{N}}\left(u_{N}^{j}, w_{N}^{i}\right) / u_{N}^{j} \vee w_{N}^{i}\right] / x_{N}\right\}
\end{gathered}
$$

where

$$
\begin{aligned}
F_{x_{l}}\left(u_{l}^{j}, w_{l}^{i}\right) & =h\left[f_{x_{l}}\left(u_{l}^{j}\right), g_{x_{l}}\left(w_{l}^{i}\right)\right] \\
& =f_{x_{l}}\left(u_{l}^{j}\right) \star g_{x_{l}}\left(w_{l}^{i}\right)
\end{aligned}
$$

and $\star$ is a $t$-norm (e.g., minimum, product, etc.). Equation (28) can also be expressed as

$$
\begin{aligned}
\tilde{A} \cup \tilde{B} & \sum_{j=1}^{M_{1}\left(u_{1}\right)} \sum_{i=1}^{M_{1}\left(w_{1}\right)}\left[F_{x_{1}}\left(u_{1}^{j}, w_{1}^{i}\right) / u_{1}^{j} \vee w_{1}^{i}\right] / x_{1}+\cdots \\
& +\sum_{j=1}^{M_{N}\left(u_{N}\right)} \sum_{i=1}^{M_{N}\left(w_{N}\right)}\left[F_{x_{N}}\left(u_{N}^{j}, w_{N}^{i}\right) / u_{N}^{j} \vee w_{N}^{i}\right] / x_{N} .
\end{aligned}
$$

Comment 5: Equation (28) is the wavy-slice expression for $\tilde{A} \cup \tilde{B}$, and although it is very easy to derive, as we demonstrate in the proof below, it is not recommended for computing purposes because it can contain an enormous number of terms. Equation (30), on the other hand, which is a vertical-slice expression for $\tilde{A} \cup \tilde{B}$, is very practical for computing purposes.

Proof: Substitute (27) into (23) to obtain (28). Equation (29) follows from Lemma 1 applied to $F_{x_{l}}\left(u_{l}^{j}, w_{l}^{i}\right)=$ $h\left[f_{x_{l}}\left(u_{l}^{j}\right), g_{x_{l}}\left(w_{l}^{i}\right)\right]$. To obtain (30), we first rewrite (28) as

$$
\begin{aligned}
\tilde{A} \cup \tilde{B} & =\sum_{j=1}^{n_{A}} \sum_{i=1}^{n_{B}}\left[F_{x_{1}}\left(u_{1}^{j}, w_{1}^{i}\right) / u_{1}^{j} \vee w_{1}^{i}\right] / x_{1}+\cdots \\
& +\sum_{j=1}^{n_{A}} \sum_{i=1}^{n_{B}}\left[F_{x_{N}}\left(u_{N}^{j}, w_{N}^{i}\right) / u_{N}^{j} \vee w_{N}^{i}\right] / x_{N} .
\end{aligned}
$$

Doing this has immediately led to a vertical-slice representation for $\tilde{A} \cup \tilde{B}$. All that remains is to simplify the upper limits in each of the summations of (31). This is very easy to do. Consider the first term in (31). According to Definition 6, $n_{A}=$ $\prod_{i=1}^{N} M_{i}\left(u_{i}\right)$ and $n_{B}=\prod_{i=1}^{N} M_{i}\left(w_{i}\right)$; but, at $x_{1}$ there are at 
most (e.g., see (6)) $M_{1}\left(u_{1}\right)$ terms from $\tilde{A}$ and $M_{1}\left(w_{1}\right)$ terms from $\tilde{B}$. Hence, we are permitted to simplify $n_{A}$ to $M_{1}\left(u_{1}\right)$ and $n_{B}$ to $M_{1}\left(w_{1}\right)$ in this first term. In the second term of (31), we are permitted to simplify $n_{A}$ to $M_{2}\left(u_{2}\right)$ and $n_{B}$ to $M_{2}\left(w_{2}\right)$. Continuing in this manner, we obtain (30).

Comment 6: Here, we connect our result in (30) with the already existing formula for the union of two type-2 fuzzy sets, given in [34]. Because the union of $\tilde{A}$ and $\tilde{B}$ is another type-2 fuzzy set, it follows from the discrete version of the first expression in (5) that:

$$
\tilde{A} \cup \tilde{B} \Leftrightarrow \mu_{\tilde{A} \cup \tilde{B}}(x, v)=\sum_{x \in X} \mu_{\tilde{A} \cup \tilde{B}}(x) / x
$$

where, from the Extension Principle [34], one obtains the following expression for $\mu_{\tilde{A} \cup \tilde{B}}(x)$ [a derivation of (33) is given in Appendix A so that readers who are unfamiliar with the application of the Extension Principle to the derivation of (32) can see exactly how it is done]:

$$
\mu_{\tilde{A} \cup \tilde{B}}(x)=\sum_{u \in J_{x}^{u}} \sum_{w \in J_{x}^{w}} f_{x}(u) \star g_{x}(w) /(u \vee w) \quad x \in X .
$$

Another way to express (33) is in terms of the secondary membership functions of $\tilde{A}$ and $\tilde{B}, \mu_{\tilde{A}}(x)$ and $\mu_{\tilde{B}}(x)$, as

$$
\begin{aligned}
\mu_{\tilde{A} \cup \tilde{B}}(x) & =\sum_{u \in J_{x}^{u}} \sum_{w \in J_{x}^{w}} f_{x}(u) \star g_{x}(w) / u \vee w \\
& \equiv \mu_{\tilde{A}}(x) \sqcup \mu_{\tilde{B}}(x) \quad x \in X
\end{aligned}
$$

where $\sqcup$ denotes the so-called join operation. The use of the notation $\mu_{\tilde{A}}(x) \sqcup \mu_{\tilde{B}}(x)$ to indicate the join between the secondary membership functions $\mu_{\tilde{A}}(x)$ and $\mu_{\tilde{B}}(x)$ is, of course, a shorthand notation for the operations in the middle of (34).

Equation (30) is exactly the same as the combination of (32) and (33), because each term of (30) is the same as (33). Whereas (33) was derived from the Extension Principle, we have been able to obtain this same result without it. Since we have reached the same results as obtained by using the Extension Principle, our results also serve to validate the use of the Extension Principle.

Note, also, that if the fourth property for $h$ is changed, then our result in (30) remains unchanged, but (33) is no longer valid because it is in terms of a $t$-norm, since the Extension Principle is in terms of a $t$-norm. In this sense, our derivation of the union is also more general than the one that uses the Extension Principle.

Comment 7: Each of the $N$ terms in (30) is a join operation, so that we can describe the union of $\tilde{A}$ and $\tilde{B}$ as the union of $N$ joins. The term "join" is very useful in that it lets us linguistically describe $\tilde{A} \cup \tilde{B}$.

\section{B. Intersection of Type-2 Fuzzy Sets}

Because the derivation of the intersection of two type-2 fuzzy sets $\tilde{A}$ and $\tilde{B}$ is so similar to the derivation of the union of those two sets, we merely state the sequence of formulas and then summarize the results in Theorem 4 whose proof we leave to the readers

$$
\begin{aligned}
\tilde{A} \cap \tilde{B}= & \sum_{j=1}^{n_{A}} \tilde{A}_{e}^{j} \cap \sum_{i=1}^{n_{B}} \tilde{B}_{e}^{i}=\sum_{j=1}^{n_{A}} \sum_{i=1}^{n_{B}} \tilde{A}_{e}^{j} \cap \tilde{B}_{e}^{i} \\
A_{e}^{j} \cap B_{e}^{i}= & u_{1}^{j} \wedge w_{1}^{i} / x_{1}+u_{2}^{j} \wedge w_{2}^{i} / x_{2}+\cdots \\
& +u_{N}^{j} \wedge w_{N}^{i} / x_{N} \\
A_{e}^{j} \cap B_{e}^{i}= & {\left[1 / u_{1}^{j} \wedge w_{1}^{i}\right] / x_{1}+\left[1 / u_{2}^{j} \wedge w_{2}^{i}\right] / x_{2}+\cdots } \\
& +\left[1 / u_{N}^{j} \wedge w_{N}^{i}\right] / x_{N} \\
\tilde{A}_{e}^{j} \cap \tilde{B}_{e}^{i} \equiv & {\left[F_{x_{1}}\left(u_{1}^{j}, w_{1}^{i}\right) / u_{1}^{j} \wedge w_{1}^{i}\right] / x_{1}+\cdots } \\
& +\left[F_{x_{N}}\left(u_{N}^{j}, w_{N}^{i}\right) / u_{N}^{j} \wedge w_{N}^{i}\right] / x_{N}
\end{aligned}
$$

Theorem 4: The intersection of two type-2 fuzzy sets $\tilde{A}$ and $\tilde{B}$ is given as

$$
\begin{gathered}
\tilde{A} \cap \tilde{B}=\sum_{j=1}^{n_{A}} \sum_{i=1}^{n_{B}}\left\{\left[F_{x_{1}}\left(u_{1}^{j}, w_{1}^{i}\right) / u_{1}^{j} \wedge w_{1}^{i}\right] / x_{1}+\cdots\right. \\
\left.+\left[F_{x_{N}}\left(u_{N}^{j}, w_{N}^{i}\right) / u_{N}^{j} \wedge w_{N}^{i}\right] / x_{N}\right\}
\end{gathered}
$$

where $F_{x_{l}}\left(u_{l}^{j}, w_{l}^{i}\right)$ is given in (29). Equation (39) can also be expressed as

$$
\begin{aligned}
& \tilde{A} \cap \tilde{B}=\sum_{j=1}^{M_{1}\left(u_{1}\right)} \sum_{i=1}^{M_{1}\left(w_{1}\right)}\left[F_{x_{1}}\left(u_{1}^{j}, w_{1}^{i}\right) / u_{1}^{j} \wedge w_{1}^{i}\right] / x_{1}+\cdots \\
& +\sum_{j=1}^{M_{N}\left(u_{N}\right)} \sum_{i=1}^{M_{N}\left(w_{N}\right)}\left[F_{x_{N}}\left(u_{N}^{j}, w_{N}^{i}\right) / u_{N}^{j} \wedge w_{N}^{i}\right] / x_{N} \text {. }
\end{aligned}
$$

Comment 8: Here, we connect our result in (40) with the already existing formula for the intersection of two type-2 fuzzy sets, given in [34]. Because the intersection of $\tilde{A}$ and $\tilde{B}$ is another type- 2 fuzzy set, it follows from the discrete version of the first expression in (5) that:

$$
\tilde{A} \cap \tilde{B} \Leftrightarrow \mu_{\tilde{A} \cap \tilde{B}}(x, v)=\sum_{x \in X} \mu_{\tilde{A} \cap \tilde{B}}(x) / x
$$

where, from the Extension Principle [34], one obtains the following expression for $\mu_{\tilde{A} \cap \tilde{B}}(x)$ [the derivation of (42) is so similar to the one given for the union that we have not included it here]:

$$
\mu_{\tilde{A} \cap \tilde{B}}(x)=\sum_{u \in J_{x}^{u}} \sum_{w \in J_{x}^{w}} f_{x}(u) \star g_{x}(w) /(u \wedge w), \quad x \in X .
$$

Another way to express (42) is in terms of the secondary membership functions of $\tilde{A}$ and $\tilde{B}, \mu_{\tilde{A}}(x)$ and $\mu_{\tilde{B}}(x)$, as

$$
\begin{aligned}
\mu_{\tilde{A} \cap \tilde{B}}(x) & =\sum_{u \in J_{x}^{u}} \sum_{w \in J_{x}^{w}} f_{x}(u) \star g_{x}(w) / u \wedge w \\
& \equiv \mu_{\tilde{A}}(x) \sqcap \mu_{\tilde{B}}(x) \quad x \in X
\end{aligned}
$$

where $\sqcap$ denotes the so-called meet operation. The use of the notation $\mu_{\tilde{A}}(x) \sqcap \mu_{\tilde{B}}(x)$ to indicate the meet between the secondary membership functions $\mu_{\tilde{A}}(x)$ and $\mu_{\tilde{B}}(x)$ is, of course, a shorthand notation for the operations in the middle of (43). 
Equation (40) is exactly the same as the combination of (41) and (42), because each term of (40) is the same as (42). Whereas (42) was derived from the Extension Principle, we have been able to obtain this same result without it. This, again, serves to validate the use of the Extension Principle. The rest of the discussion in the last paragraph of Comment 6 applies here as well.

Comment 9: Each of the $N$ terms in (40) is a meet operation, so that we can describe the intersection of $\tilde{A}$ and $\tilde{B}$ as the union of $N$ meets. The term "meet" is very useful in that it lets us linguistically describe $\tilde{A} \cap \tilde{B}$.

\section{Complement of Type-2 Fuzzy Sets}

Our final application of the Representation Theorem is to compute the complement of $\tilde{A}$.

Theorem 5: The complement of type- 2 fuzzy set $\tilde{A}$ is given as

$$
\overline{\widetilde{A}}=\sum_{j=1}^{n_{A}}\left(\sum_{i=1}^{N}\left[f_{x_{i}}\left(u_{i}^{j}\right) /\left(1-u_{i}^{j}\right)\right] / x_{i}\right)
$$

where $n_{A}$ is given by (16). Equation (44) can also be expressed as

$$
\overline{\widetilde{A}}=\sum_{i=1}^{N}\left(\sum_{j=1}^{M_{i}}\left[f_{x_{i}}\left(u_{i}^{j}\right) /\left(1-u_{i}^{j}\right)\right] / x_{i}\right) .
$$

Proof: From (15), we see that

$$
\overline{\widetilde{A}}=\sum_{j=1}^{n_{A}} \overline{\widetilde{A}}_{e}^{j}
$$

which demonstrates that to evaluate $\overline{\widetilde{A}}$ we need to evaluate the complement of type- 2 embedded sets, i.e., we need to evaluate $\overline{\tilde{A}}_{e}^{j}$. In the type-1 case

$$
\bar{A}_{e}^{j}=\left(1-u_{1}^{j}\right) / x_{1}+\cdots+\left(1-u_{N}^{j}\right) / x_{N}
$$

which can be expressed as a type-2 fuzzy set, as follows:

$$
\bar{A}_{e}^{j}=\left[1 /\left(1-u_{1}^{j}\right)\right] / x_{1}+\cdots+\left[1 /\left(1-u_{N}^{j}\right)\right] / x_{N} \text {. }
$$

We now define $\overline{\widetilde{A}}_{e}^{j}$ as follows:

$$
\begin{aligned}
\overline{\tilde{A}}_{e}^{j} \equiv & {\left[f_{x_{1}}\left(u_{1}^{j}\right) /\left(1-u_{1}^{j}\right)\right] / x_{1}+\cdots } \\
& +\left[f_{x_{N}}\left(u_{N}^{j}\right) /\left(1-u_{N}^{j}\right)\right] / x_{N} \\
= & \sum_{i=1}^{N}\left[f_{x_{i}}\left(u_{i}^{j}\right) /\left(1-u_{i}^{j}\right)\right] / x_{i} .
\end{aligned}
$$

Substituting (49) into (46), we obtain (44).

To obtain (45), we first rewrite (44) as

$$
\begin{aligned}
\overline{\tilde{A}}=\sum_{j=1}^{n_{A}}\left[f_{x_{1}}\left(u_{1}^{j}\right) /\left(1-u_{1}^{j}\right)\right] / x_{1}+\cdots \\
+\sum_{j=1}^{n_{A}}\left[f_{x_{N}}\left(u_{N}^{j}\right) /\left(1-u_{N}^{j}\right)\right] / x_{N} .
\end{aligned}
$$

Using exactly the same arguments that we did at the end of the proof of Theorem 3, we conclude that (50) can be re-expressed as in (45).

Comment 10: Because the complement of $\tilde{A}$ is another type-2 fuzzy set, it follows again from the discrete version of the first expression in (5) that:

$$
\overline{\widetilde{A}} \Leftrightarrow \mu_{\bar{A}}(x, v)=\sum_{x \in X} \mu_{\tilde{\tilde{A}}}(x) / x
$$

where, from the Extension Principle [34], one obtains the following expression for $\mu_{\bar{A}}$ [we leave the derivation of (52) to the reader]:

$$
\mu_{\tilde{A}}(x)=\sum_{u \in J_{x}^{u}} f_{x}(u) /(1-u) \equiv \neg \mu_{\tilde{A}}(x) \quad x \in X
$$

in which $\neg$ denotes the so-called negation operation. The use of the notation $\neg \mu_{\tilde{A}}(x)$ to indicate the negation of the secondary membership function $\mu_{\tilde{A}}(x)$ is yet another shorthand notation, but this time for the operations in the middle of (52).

Equation (45) is exactly the same as the combination of (51) and (52), because each term of (45) is the same as (52). Whereas (52) was derived from the Extension Principle, we have been able to obtain this same result without it, which again serves to validate the use of the Extension Principle.

Comment 11: Each of the $N$ terms in (45) is a negation operation, so that we can describe the complement of $\tilde{A}$ as the union of $N$ negations. The term "negation" is very useful in that it lets us linguistically describe $\tilde{A}$.

\section{Interval Type-2 Fuzzy Sets}

Interval type-2 fuzzy sets are the most widely used type-2 fuzzy sets because they are simple to use and because, at present, it is very difficult to justify the use of any other kind (e.g., there is no best choice for a type-1 fuzzy set, so to compound this nonuniqueness by leaving the choice of the secondary membership functions arbitrary is hardly justifiable ${ }^{9}$ ). When the type-2 fuzzy sets are interval type-2 fuzzy sets, all secondary grades (flags) equal 1 [e.g., in (21) and (22), $\forall f_{x_{1}}\left(u_{l}^{j}\right)=1$ and $\left.\forall g_{x_{l}}\left(w_{l}^{i}\right)=1\right]$. In this case we can treat embedded type-2 fuzzy sets as embedded type-1 fuzzy sets [e.g., (27) is the same as (25)] so that no new concepts are needed to derive the union, intersection, and complement of such sets. After each derivation, we merely append interval secondary grades to all the results in order to obtain the final formulas for the union, intersection, and complement of interval type- 2 fuzzy sets. Closed-form formulas exist for these operations, and their derivations can be found, e.g., in [33, Ch.. 7].

\section{CONCLUSiON}

We have established a small set of terms (type-2 membership function, secondary membership function, vertical-slice, primary membership, secondary grade, footprint of uncertainty, embedded type-2 fuzzy set, embedded type-1 fuzzy set, and wavy-slice) that let us easily communicate about type-2 fuzzy sets. They also let us define such sets very precisely. We have

\footnotetext{
${ }^{9}$ This is analogous to using a uniform probability density function (pdf) when all that is known is that something is random, but the precise nature of the underlying pdf is unknown.
} 
also presented a new Representation Theorem for type-2 fuzzy sets and have shown how it can be used to derive formulas for the union, intersection, and complement of type-2 fuzzy sets without having to use the Extension Principle. In doing so , we believe that we have made type-2 fuzzy sets much easier to understand and to work with, which was our stated goal.

The centroid of a type-2 fuzzy set has been defined by [15] and $[19]^{10}$. All centroid formulas start with a formula for the centroid of a type-1 fuzzy set to which the Extension Principle is applied. Computational procedures are then provided for actually computing the centroid of a type- 2 fuzzy set. These procedures all wind up computing centroids of all possible embedded type-2 fuzzy sets that are associated with the original type-2 fuzzy set. It is the totality of all such centroids that make up the centroid of the type-2 fuzzy set. Using the new Representation Theorem of this paper, we could immediately define the centroid of a type-2 fuzzy set, in a very rigorous way, as the centroid of all of its embedded type-2 fuzzy sets. By this approach, we reach exactly the same result as was reached by invoking the Extension Principle, but without having to use it.

We await other applications of our Representation Theorem.

\section{APPENDIX A}

\section{Derivation of the Union of Two Type-2 FuZzy Sets USING THE EXTENSION PRINCIPLE}

In this Appendix, we review the statement of the Extension Principle and present a version of it that we then use to obtain the union of two type-2 fuzzy sets. We leave the derivations of the intersection of two type-2 fuzzy sets as well as the complement of a type-2 fuzzy set to the reader.

\section{A. The Extension Principle}

Let $A_{1}, A_{2}, \ldots, A_{r}$ be type- 1 fuzzy sets in $X_{1}, X_{2}, \ldots, X_{r}$, respectively. Then, Zadeh's Extension Principle [44] allows us to induce from the $r$ type-1 fuzzy sets $A_{1}, A_{2}, \ldots, A_{r}$ a type-1 fuzzy set $B$ on $Y$, through $f$, i.e., $B=f\left(A_{1}, A_{2}, \ldots, A_{r}\right)$, such that

$$
\mu_{B}(y)=\left\{\begin{array}{l}
\sup _{\left(x_{1}, \ldots, x_{r}\right) \in f^{-1}(y)} \min \left\{\mu_{A_{1}}\left(x_{1}\right), \ldots, \mu_{A_{r}}\left(x_{r}\right)\right\} \\
0 \quad \text { if } f^{-1}(y)=\emptyset
\end{array}\right.
$$

where $f^{-1}(y)$ denotes the set of all points $x_{1} \in X_{1}, \ldots, x_{r} \in$ $X_{r}$ such that $f\left(x_{1}, \ldots, x_{r}\right)=y$.

As is well known, to implement (A-1) we first find the values of $x_{1}, \ldots, x_{r}$ for which $y=f\left(x_{1}, \ldots, x_{r}\right)$, after which we compute $\mu_{A_{1}}\left(x_{1}\right), \ldots, \mu_{A_{r}}\left(x_{r}\right)$ and $\min \left\{\mu_{A_{1}}\left(x_{1}\right), \ldots, \mu_{A_{r}}\left(x_{r}\right)\right\}$ at those values. If more than one set of $x_{1}, \ldots, x_{r}$ satisfy $y=f\left(x_{1}, \ldots, x_{r}\right)$ then we repeat this procedure for all of them and choose the largest of the minima as the choice for $\mu_{B}(y)$.

Zadeh defined the Extension Principle using minimum $t$-norm and maximum $t$-conorm (for the supremum operation). Other $t$-norms and $t$-conorms can be used as described, e.g., in [34] and [6].

\footnotetext{
${ }^{10}$ See, also [33]
}

When we need to extend an operation of the form $f\left(x_{1}, \ldots, x_{r}\right)$ to an operation $f\left(A_{1}, \ldots, A_{r}\right)$, where the $A_{i}$ are type- 1 fuzzy sets, we do not extend the individual operations, like multiplication, addition, etc., involved in $f$. Instead, we use the following definition, which derives directly from (A-1) when the maximum operation is used for the union and a general $t$-norm $(\star)$ is used instead of the minimum operation

$$
\begin{aligned}
& f\left(A_{1}, \ldots, A_{r}\right)=\int_{x_{1} \in X_{1}} \cdots \int_{x_{r} \in X_{r}} \mu_{A_{1}}\left(x_{1}\right) \star \cdots \\
& \star \mu_{A_{r}}\left(x_{r}\right) / f\left(x_{1}, \ldots, x_{r}\right) \text {. }
\end{aligned}
$$

\section{B. Derivation of the Union of Two Type-2 Fuzzy Sets}

Using a discrete version of (5), (32) can be re-expressed as

$$
\begin{aligned}
\tilde{A} \cup \tilde{B} & \Leftrightarrow \mu_{\tilde{A} \cup \tilde{B}}(x, v)=\sum_{x \in X} \mu_{\tilde{A} \cup \tilde{B}}(x) / x \\
& =\sum_{x \in X}\left[\sum_{v \in J_{x}^{v} \subseteq[0,1]} h_{x}(v) / v\right] / x
\end{aligned}
$$

where

$$
\begin{aligned}
\sum_{v \in J_{x}^{v} \subseteq[0,1]} h_{x}(v) / v & =\varphi\left(\sum_{u \in J_{x}^{u}} f_{x}(u) / u, \sum_{w \in J_{x}^{w}} g_{x}(w) / w\right) \\
& =\varphi\left(\mu_{\tilde{A}}(x), \mu_{\tilde{B}}(x)\right)
\end{aligned}
$$

and $\varphi$, which plays the role of $f$ in (A-2), is a $t$-conorm function of the secondary membership functions, $\mu_{\tilde{A}}(x)$ and $\mu_{\tilde{B}}(x)$, which are type- 1 fuzzy sets. $\varphi$ is a $t$-conorm function because the union of two type- 1 fuzzy sets is equivalent to the $t$-conorm (e.g., maximum) of their membership functions. Note that the right-hand side of (A-4) plays the role of $f\left(x_{1}, x_{2}\right)$ in the Extension Principle. Following the prescription of the right-hand side of (A-2), we see that:

$$
\begin{aligned}
\varphi\left(\sum_{u \in J_{x}^{u}} f_{x}(u) / u\right. & \left., \sum_{w \in J_{x}^{w}} g_{x}(w) / w\right) \\
& =\sum_{u \in J_{x}^{u}} \sum_{w \in J_{x}^{w}} f_{x}(u) \star g_{x}(w) / \varphi(u, w) .
\end{aligned}
$$

When $\varphi$ is the maximum operation $\vee$, then $\varphi(u, w)=u \vee w$, so that when (A-5) is substituted into (A-3) for $\mu_{\tilde{A} \cup \tilde{B}}(x)$ we obtain

$$
\begin{aligned}
\mu_{\tilde{A} \cup \tilde{B}}(x) & =\sum_{v \in J_{x}^{v} \subseteq[0,1]} h_{x}(v) / v \\
& =\sum_{u \in J_{x}^{u}} \sum_{w \in J_{x}^{w}} f_{x}(u) \star g_{x}(w) /(u \vee w) \quad x \in X
\end{aligned}
$$

which is (33).

\section{REFERENCES}

[1] H. Bustince and P. Burillo, "Mathematical analysis of interval-valued fuzzy relations: Application to approximate reasoning," Fuzzy Sets Syst., vol. 113, pp. 205-219, 2000

[2] J. L. Chaneau, M. Gunaratne, and A. G. Altschaeffl, "An application of type-2 sets to decision making in engineering," in Analysis of Fuzzy Information, vol. II: Artificial Intelligence and Decision Systems, J. Bezdek, Ed. Boca Raton, FL: CRC Press, 1987. 
[3] D. A. Chiang, L.-R. Chow, and N.-C. Hsien, "Fuzzy information in extended fuzzy relational databases," Fuzzy Sets Syst., vol. 92, pp. 1-20, Nov. 1997.

[4] D. Dubois and H. Prade, "Operations on fuzzy numbers," Int. J. Syst. Sci., vol. 9, pp. 613-626, 1978.

[5] — , "Operations in a fuzzy-valued logic," Inform. Control, vol. 43, pp. 224-240, 1979

[6] _ - Fuzzy Sets and Systems: Theory and Applications. New York: Academic, 1980.

[7] M. B. Gorzalczany, "A method of inference in approximate reasoning based on interval-valued fuzzy sets," Fuzzy Sets Syst., vol. 21, pp. 1-17, 1987.

[8] E. Hisdal, "The IF THEN ELSE statement and interval-values fuzzy sets of higher type," Int. J. Man-Machine Studies, vol. 15, pp. 385-455, 1981.

[9] K. Izumi, H. Tanaka, and K. Asai, "Resolution of composite fuzzy relational equations of type 2," Trans. Inst. Electr. Commun. Engineers Japan, pt. D, vol. J66D, pp. 1107-1113, Oct. 1983. in Japanese.

[10] R. I. John, "Type-2 inferencing and community transport scheduling," in Proc. 4th Euro. Congress Intelligent Techniques Soft Computing, Aachen, Germany, Sept. 1996, pp. 1369-1372.

[11] — " "Type 2 fuzzy sets for knowledge representation and inferencing," Proc. IEEE Int. Conf. Fuzzy Systems, IEEE World Congress Computational Intelligence, pp. 1003-1008, May 1998.

[12] R. I. John and C. Czarnecki, "An adaptive type-2 fuzzy system for learning linguistic membership grades," Proc. 8th Int. Conf. Fuzzy Systems, pp. 1552-1556, Aug. 1999.

[13] R. I. John, P. R. Innocent, and M. R. Barnes, "Type-2 fuzzy sets and neuro-fuzzy clustering or radiographic tibia images," in Proc.6th Int. Conf. on Fuzzy Systems, Barcelona, Spain, July 1997, pp. 1375-1380.

[14] N. N. Karnik and J. M. Mendel, "Introduction to type-2 fuzzy logic systems," Proc. 1998 IEEE FUZZ Conf., pp. 915-920, May 1998.

[15] — (1998b, June) An Introduction to Type-2 Fuzzy Logic Systems. Univ. of Southern Calif., Los Angeles, CA. [Online]. Available: http://sipi.usc.edu/ mendel/report

[16] — "Type-2 fuzzy logic systems: Type-reduction," Proc. IEEE Conference on Systems, Man and Cybernetics, pp. 2046-2051, Oct. 1998c.

[17] _- "Applications of type-2 fuzzy logic systems to forecasting of timeseries," Information Sciences, vol. 120, pp. 89-111, 1999a.

[18] — , "Operations on type-2 fuzzy sets," Int. J. Fuzzy Sets Syst., vol. 122, pp. 327-348, 2001.

[19] — "Centroid of a type-2 fuzzy set," Inform. Sci., vol. 132, pp. 195-220, 2001

[20] N. N. Karnik, J. M. Mendel, and Q. Liang, "Type-2 fuzzy logic systems," IEEE Trans. Fuzzy Syst., vol. 7, pp. 643-658, Dec. 1999.

[21] V. Kreinovich, A. Lakeyev, J. Rohn, and P. Kahl, Computational Complexity and Feasibility of Data Processing and Interval Computations, MA, Boston: Kluwer, 1998, ch. 10.

[22] R. N. Lea, V. Kreinovich, and R. Trejo, "Optimal interval enclosures for fractionally-linear functions and their application to intelligent control," Rel. Comput., vol. 2, pp. 265-285, 1996.

[23] Q. Liang and J. M. Mendel, "An introduction to type-2 TSK fuzzy logic systems," Proc. FUZZ-IEEE, 1999.

[24] — , "Interval type-2 fuzzy logic systems," Proc. FUZZ-IEEE, May 2000.

[25] — "Decision feedback equalizer for nonlinear time-varying channels using type-2 fuzzy adaptive filters," Proc. FUZZ-IEEE, May 2000.

[26] _ - "Interval type-2 fuzzy logic systems: Theory and design," IEEE Trans. Fuzzy Syst., vol. 8, pp. 535-550, Oct. 2000.

[27] — - "Equalization of nonlinear time-varying channels using type-2 fuzzy adaptive filters," IEEE Trans. Fuzzy Syst., vol. 8, pp. 551-563, Oct. 2000

[28] —, "Overcoming time-varying co-channel interference using type-2 fuzzy adaptive filter," IEEE Trans. Circuits Syst.-II: Analog and Digital Signal Processing, vol. 47, pp. 1419-1428, Dec. 2000.

[29] — , "MPEG VBR video traffic modeling and classification using fuzzy techniques," IEEE Trans. Fuzzy Syst., vol. 9, pp. 183-193, Feb. 2001.

[30] Q. Liang, N. N. Karnik, and J. M. Mendel, "Connection admission control in ATM networks using survey-based type-2 fuzzy logic systems," IEEE Trans. Syst., Man, Cybern. Part C: Appl. Reviews, vol. 30, pp. 329-339, 2000.
[31] S. Mabuchi, "An interpretation of membership functions and the properties of general probabilistic operators as fuzzy set operators, II: Extension to three-valued and interval-valued fuzzy sets," Fuzzy Sets Syst. vol. 92, pp. 31-50, Nov. 1997.

[32] J. M. Mendel, "Uncertainty, fuzzy logic, and signal processing," Signal Proc. J., vol. 80, pp. 913-933, 2000.

[33] — Uncertain Rule-Based Fuzzy Logic Systems: Introduction and New Directions. Upper Saddle River, NJ: Prentice-Hall, 2001.

[34] M. Mizumoto and K. Tanaka, "Some properties of fuzzy sets of type-2," Inform. Control, vol. 31, pp. 312-340, 1976

[35] _ - "Fuzzy sets of type-2 under algebraic product and algebraic sum," Fuzzy Sets Syst., vol. 5, pp. 277-290, 1981.

[36] H. T. Nguyen, V. Kreinovich, and Q. Zuo, "Interval-valued degrees of belief: Applications of interval computations to expert systems and intelligent control," Int. J. Uncertainty, Fuzziness, Knowledge-Based Syst., vol. 5, pp. 317-358, 1997.

[37] J. Nieminen, "On the algebraic structure of fuzzy sets of type-2," Kybernetica, vol. 13, no. 4, 1977 .

[38] D. G. Schwartz, "The case for an interval-based representation of linguistic truth," Fuzzy Sets Syst., vol. 17, pp. 153-165, 1985.

[39] M. Sugeno, "Fuzzy theory, III," J. Soc. Instrum. Control Eng., vol. 22, pp. 454-458, May 1983. in Japanese.

[40] I. Turksen, "Interval valued fuzzy sets based on normal forms," Fuzzy Sets Syst., vol. 20, pp. 191-210, 1986.

[41] M. Wagenknecht and K. Hartmann, "Application of fuzzy sets of type-2 to the solution of fuzzy equation systems," Fuzzy Sets Syst., vol. 25, pp. $183-190,1988$

[42] K. C. Wu, "Fuzzy interval control of mobile robots," Comput. Elect. Eng., vol. 22, pp. 211-229, 1996.

[43] R. R. Yager, "Fuzzy subsets of type II in decisions," J. Cybern., vol. 10, pp. $137-159,1980$

[44] L. A. Zadeh, "The concept of a linguistic variable and its application to approximate reasoning - 1," Inform. Sci., vol. 8, pp. 199-249, 1975.

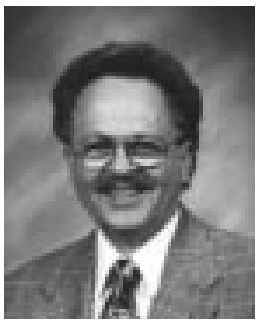

Jerry M. Mendel received the Ph.D. degree in electrical engineering from the Polytechnic Institute of Brooklyn, NY.

Since 1974, he has been Professor of Electrical Engineering, and, since 1996, Associate Director for Education of the Integrated Media Systems Center at the University of Southern California, Los Angeles. He has published over 400 technical papers and is author and/or editor of eight books, including Uncertain Rule-Based Fuzzy Logic Systems: Introduction and New Directions (Upper Saddle River, NJ: Prentice-Hall, 2001). His present research interests include type-2 fuzzy logic systems and their applications to a wide range of problems.

Dr. Mendel is a Distinguished Member of the IEEE Control Systems Society. He was President of the IEEE Control Systems Society in 1986. Among his awards are the 1983 Best Transactions Paper Award of the IEEE Geoscience and Remote Sensing Society, the 1992 Signal Processing Society Paper Award, a 1984 IEEE Centennial Medal, and an IEEE Third Millenium Medal.

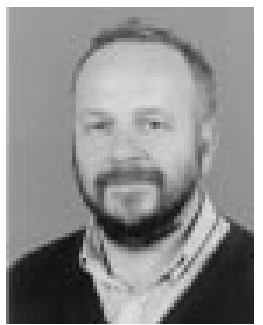

Robert I. Bob John received the B.Sc. (Hons.) degree in mathematics from Leicester Polytechnic, Leicester, U.K., the M.Sc. degree in statistics from UMIST, Manchester, U.K., and the Ph.D. degree in type-2 fuzzy logic De Montfort University, Leicester, U.K., in 1979, 1981, and 2000, respectively.

$\mathrm{He}$ is currently Director of the Centre for Computational Intelligence (CCI) at De Montfort University, Leicester, U.K. The CCI specializes in using soft computing techniques for tackling difficult problems, as well as developing theoretical ideas in fuzzy logic. He has published over one hundered papers on fuzzy logic and has edited three books. His research interests relate to the role of type-2 fuzzy logic in modeling perceptions and, in particular, for modeling medical expertise. He has developed automatic learning for type-2 fuzzy logic systems. 